\title{
Hepatic stellate cells in liver development, regeneration, and cancer
}

\author{
Chunyue Yin, ${ }^{1}$ Kimberley J. Evason, ${ }^{2}$ Kinji Asahina, ${ }^{3}$ and Didier Y.R. Stainier ${ }^{1}$
}

\begin{abstract}
${ }^{1}$ Department of Biochemistry and Biophysics, Programs in Developmental and Stem Cell Biology, Genetics and Human Genetics, Liver Center and Diabetes Center, Institute for Regeneration Medicine, and 2Department of Pathology, UCSF, San Francisco, California, USA. ${ }^{3}$ Southern California Research Center for ALPD and Cirrhosis and Department of Pathology, Keck School of Medicine of the University of Southern California, Los Angeles, California, USA.
\end{abstract}

\begin{abstract}
Hepatic stellate cells are liver-specific mesenchymal cells that play vital roles in liver physiology and fibrogenesis. They are located in the space of Disse and maintain close interactions with sinusoidal endothelial cells and hepatic epithelial cells. It is becoming increasingly clear that hepatic stellate cells have a profound impact on the differentiation, proliferation, and morphogenesis of other hepatic cell types during liver development and regeneration. In this Review, we summarize and evaluate the recent advances in our understanding of the formation and characteristics of hepatic stellate cells, as well as their function in liver development, regeneration, and cancer. We also discuss how improved knowledge of these processes offers new perspectives for the treatment of patients with liver diseases.
\end{abstract}

Hepatic stellate cells are located in the space of Disse between the sinusoidal endothelial cells and hepatic epithelial cells, and account for $5 \%-8 \%$ of the cells in the liver. In a healthy liver, stellate cells are quiescent and contain numerous vitamin A lipid droplets, constituting the largest reservoir of vitamin A in the body (reviewed in ref. 1). When the liver is injured due to viral infection or hepatic toxins, hepatic stellate cells receive signals secreted by damaged hepatocytes and immune cells, causing them to transdifferentiate into activated myofibroblastlike cells (reviewed in ref. 2). As the primary extracellular matrixproducing (ECM-producing) cells in liver, activated stellate cells generate a temporary scar at the site of injury to protect the liver from further damage. In addition, hepatic stellate cells secrete cytokines and growth factors that promote the regeneration of hepatic epithelial cells. In chronic liver disease, prolonged and repeated activation of stellate cells causes liver fibrosis, as characterized by widespread scar formation and perturbation of liver architecture and function (reviewed in ref. 3). Recent clinical and experimental evidence indicates that hepatic fibrosis is reversible upon removal of the underlying etiological agent (4-6). During the regression of liver fibrosis, the number of activated hepatic stellate cells is greatly reduced by the induction of cellular senescence and apoptosis, or by the return to the quiescent state $(2,5-7)$. Because of their pivotal roles in liver repair and disease pathogenesis, hepatic stellate cells have been a major focus of liver research. However, our knowledge of their cell biology is far from complete, mainly due to the challenges of studying these cells in vivo.

This Review focuses on the recent insights and emerging investigations into the formation of hepatic stellate cells and their function in liver development, regeneration, and hepatocellular carcinoma (HCC). The regulation of stellate cells in liver fibrosis as well as the design of antifibrotic therapies is reviewed separately in this issue (8).

Authorship note: Chunyue Yin and Kimberley J. Evason contributed equally to this work.

Conflict of interest: The authors have declared that no conflict of interest exists. Citation for this article: J Clin Invest. 2013;123(5):1902-1910. doi:10.1172/JCI66369.

\section{Experimental models to study hepatic stellate cells}

Over the past two decades, the development of cell culture system and genetic animal models (summarized in Figure 1) has greatly advanced our understanding of the cellular properties of hepatic stellate cells and their function in healthy as well as injured livers. When cultured on plastic, freshly isolated hepatic stellate cells undergo spontaneous activation (9-11). This cell culture system, along with other hepatic stellate cell lines (12-14), recapitulates many aspects of hepatic stellate cell activation in vivo. But hepatic stellate cells activated in culture do not fully reproduce the changes in gene expression observed in vivo, making it difficult in some cases to correlate in vitro results with hepatic stellate cell behaviors in vivo (15).

In the animal, hepatic stellate cells can be identified based on expression of desmin (16) and glial fibrillary acidic protein (GFAP) (17) in the quiescent state and $\alpha$-SMA in the activated state (18). The identification of promoters that selectively drive transgene expression in hepatic stellate cells might facilitate both in vivo observations and genetic manipulation of these cells. Components of collagen $\alpha 1(\mathrm{I})$, collagen $\alpha 2(\mathrm{I})$, and $\alpha S M A$ promoters were used to direct reporter gene expression in activated hepatic stellate cells in transgenic mice (19). Promoter elements of the $\operatorname{Gfap}(20,21)$ and vimentin (6) genes drive gene expression in quiescent hepatic stellate cells. However, neither promoter is specific for hepatic stellate cells: Gfap promoter activity is detected in neuronal tissues and cholangiocytes (21), whereas the vimentin gene is also expressed in vascular smooth muscle cells and portal fibroblasts (6).

The zebrafish has emerged as a valuable vertebrate model system to study liver development and disease. The rapid external development and translucence of zebrafish embryos and larvae make them well suited for in vivo imaging $(22,23)$. The availability of transgenic lines that express fluorescent proteins in different hepatic cell types allows easy visualization of cell behaviors in the animal and greatly facilitates genetic and chemical screens to identify regulators of liver development and disease pathogenesis. Our group recently reported a transgenic zebrafish line, $\mathrm{Tg}$ (hand2:EGFP), that expresses EGFP under the promoter of the hand2 gene (24). The transgene expression marks both quiescent and activated hepatic stellate cells. Zebrafish hepatic stellate cells 
Quiescent hepatic stellate cells
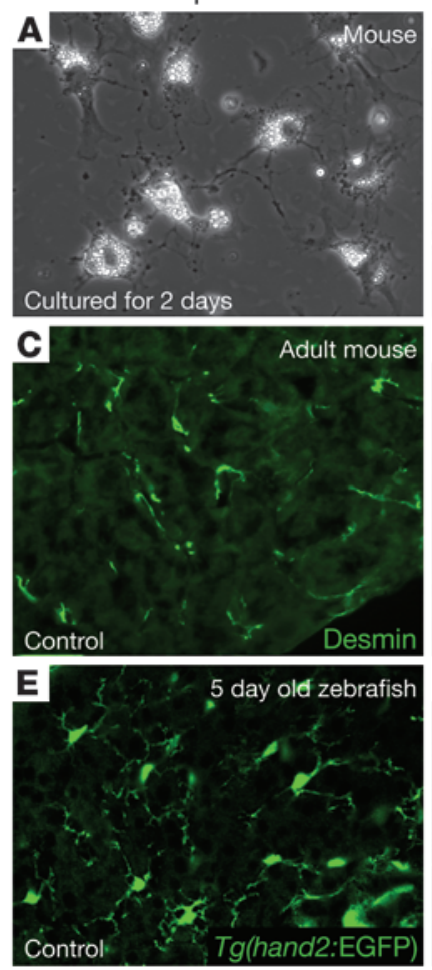

Activated hepatic stellate cells
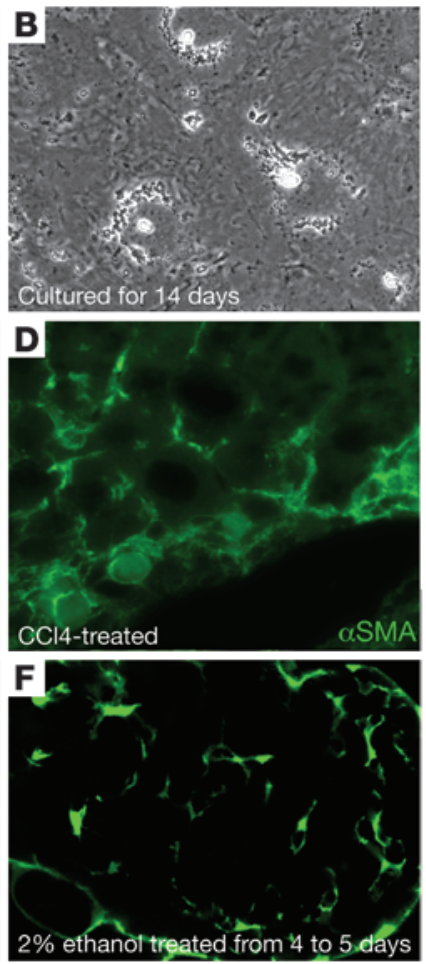

exhibit all the hallmarks of mammalian hepatic stellate cells, including morphology, localization, vitamin A storage, and gene expression profile. Significantly, zebrafish hepatic stellate cells become activated in response to an acute alcohol insult, as evidenced by increased proliferation and ECM production (Figure 1 and ref. 24). This zebrafish hepatic stellate cell reporter line thus represents a novel animal model that complements the cell culture and mammalian model systems.

\section{Hepatic stellate cells in liver development}

Knowledge about the characteristics, lineage, and function of stellate cells during liver development is critical to obtaining a fundamental understanding of hepatic stellate cell activation and their role in liver diseases. Recent studies in animal models and cell culture systems have provided key insights regarding hepatic stellate cells during development, but important gaps remain in our knowledge of this process.

Embryonic origin of hepatic stellate cells. The embryonic origin of hepatic stellate cells is unresolved because they express marker genes of all three germ layers (reviewed in ref. 2). Lineage tracing of the Wilms tumor suppressor gene-expressing (Wt1-expressing) cells and mesoderm posterior 1-expressing cells in mice showed that hepatic stellate cells develop from the septum transversum-derived mesothelium lining the liver $(25,26)$, suggestive of a mesodermal origin. On the other hand, stellate cells in the human fetal liver express CD34 and cytokeratin-7/8, connecting them to an endodermal origin $(27,28)$. Along this theme, hepatic epithelial cells are thought to transdifferentiate into hepatic stellate cells in the injured liver through epithelial-mesenchymal transition (EMT) (29). However, the contribution of EMT to the hepatic stellate cell lineage is highly controversial (30). Lastly, bone marrow-derived mesenchymal cells are also thought to

\section{Figure 1}

Models for studying hepatic stellate cells. (A) Phase contrast image of mouse hepatic stellate cells cultured for 2 days. These hepatic stellate cells are still quiescent, as evidenced by their vitamin A lipid deposition, a stellate morphology, and presence of dendritic processes. (B) Phase contrast image of mouse hepatic stellate cells cultured for 14 days. By this time, hepatic stellate cells are fully activated and exhibit dramatic changes in their morphology and reduction in lipid deposition. (C) Fluorescence image of hepatic stellate cells in healthy adult mouse liver stained for desmin. (D) Fluorescence image shows $\alpha$-SMA immunostaining in $\mathrm{CCl}_{4}$-induced fibrosis in the adult mouse liver. (E) Confocal single-plane image of $T g$ (hand2:EGFP) expression in zebrafish hepatic stellate cells at 5 days after fertilization. The hepatic stellate cells exhibit a stellate morphology and send out complex protrusions (23). (F) Confocal single-plane image of hepatic stellate cells labeled by $\operatorname{Tg}$ (hand2:EGFP) expression in zebrafish larvae treated with $2 \%$ ethanol from 4 to 5 days after fertilization. Hepatic stellate cells become activated upon the acute ethanol assault, as evidenced by the loss of complex cellular processes and elongated cell body, suggestive of changes in contractility (24).

contribute to both quiescent and activated hepatic stellate cells $(31,32)$, although several reports indicate that this contribution is negligible $(33,34)$.

It is noteworthy that in mice, the septum transversum-derived mesothelial cells give rise not only to hepatic stellate cells, but also to perivascular mesenchymal cells, including portal fibroblasts, smooth muscle cells around the portal vein, and fibroblasts around the central vein (26). Following liver injury, activated stellate cells are the major source of myofibroblasts. However, portal fibroblasts and vascular myofibroblasts can also become myofibroblasts, but their contribution to fibrogenesis might be different from the hepatic stellate cell-derived myofibroblasts $(35,36)$. Therefore, an understanding of how the cell fate decision is made between hepatic stellate cells and perivascular mesenchymal cells might aid in the design of therapies to specifically target hepatic stellate cells.

In both fetal and adult livers, stellate cells are closely associated with sinusoidal endothelial cells, which also derive from mesoderm. Because of their physical proximity and shared expression of angiogenic factors (37), hepatic stellate cells and sinusoidal endothelial cells have been proposed to share a common precursor. This hypothesis is supported by observations in chick embryos that the mesothelium contributes to both cell populations (38). In zebrafish, however, stellate cells are still present in the liver of cloche mutants that lack sinusoidal endothelial cells and their precursors (24). This result indicates that neither endothelial cells nor their precursors are required for hepatic stellate cell differentiation or their entry into the liver.

Regulation of hepatic stellate cell development. To date, only a few studies have addressed early hepatic stellate cell behaviors in vivo. Tracking of the Wt1-expressing septum transversum cells in mice showed that these cells migrate inward from the liver surface while differentiating into hepatic stellate cells (ref. 25 and see Figure 2A). A similar migration behavior of hepatic stellate cells was observed in zebrafish (24). Furthermore, the migration of septum transversum cells from the liver surface likely constitutes the main source of new stellate cells during zebrafish development, as they rarely proliferate after entering the liver.

Studies in mutant mice have revealed the roles of several mesenchymal-specific genes in hepatic stellate cell development (summarized in Figure 2A). Wt1 and the LIM homeobox gene $L b x 2$ are 
A

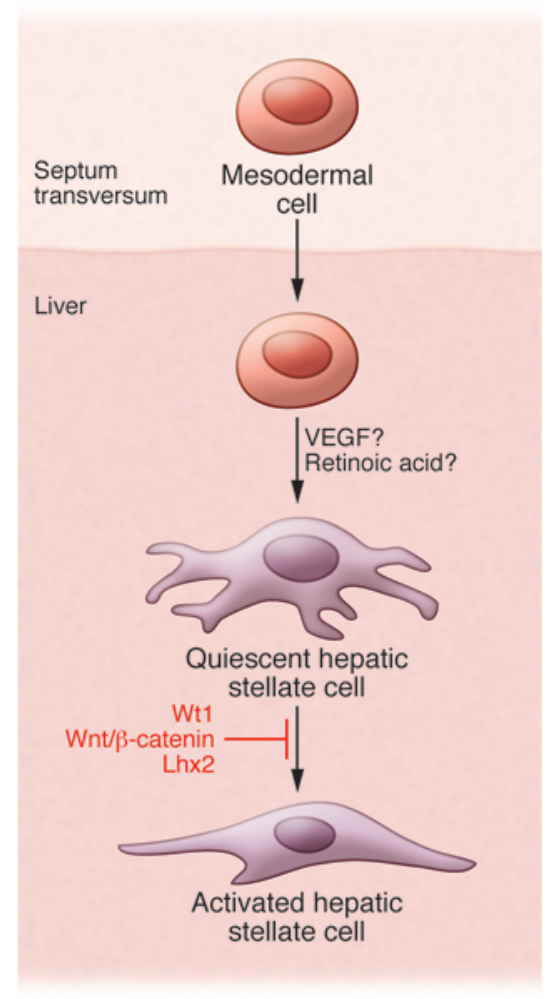

B

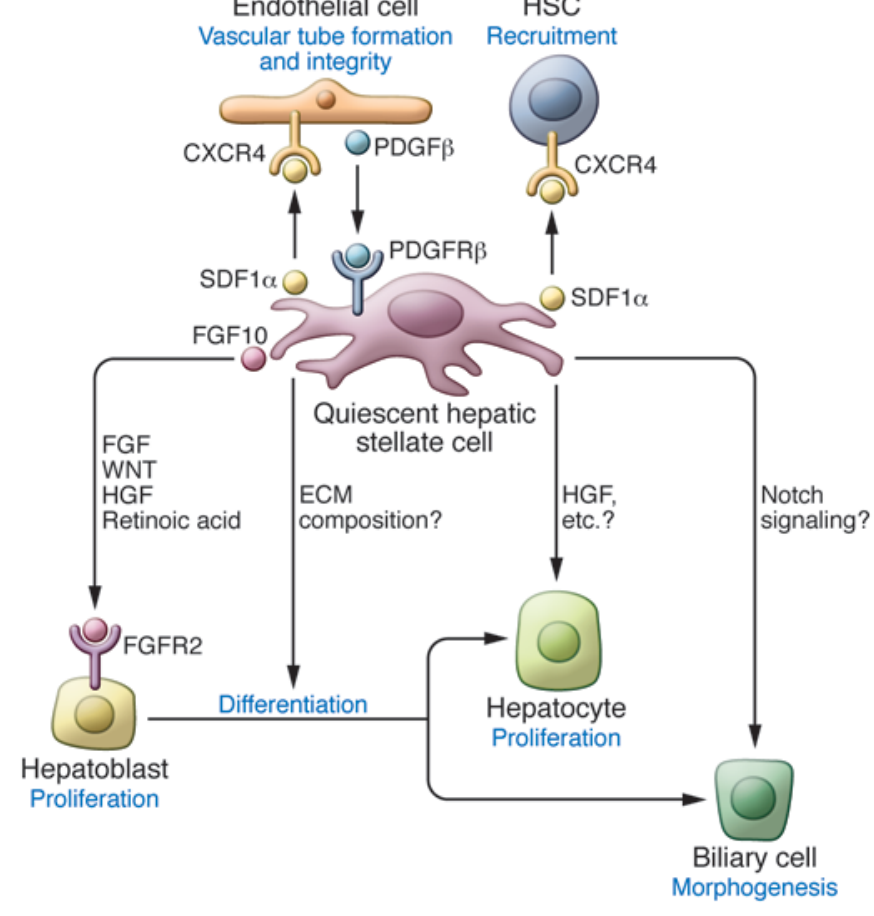

Figure 2

Hepatic stellate cell development and contribution to liver organogenesis. (A) Hepatic stellate cell development. Lineage-tracing analyses in mice indicate that during development, the mesodermal cells within the septum transversum invade the liver while differentiating into hepatic stellate cells and perivascular mesenchymal cells. VEGF and retinoic acid signaling are both required for hepatic stellate cell formation, potentially affecting the migration of septum transversum cells, the differentiation of hepatic stellate cells, or both. Wt1, Wnt/ $\beta$-catenin signaling, and Lhx2 inhibit aberrant activation of hepatic stellate cells in the developing liver. (B) Contribution of hepatic stellate cells to hepatic organogenesis. The biological processes influenced by hepatic stellate cells are indicated in blue. For endothelial cells, hepatic stellate cells secrete the chemokine SDF1, whereas endothelial cells express its receptor CXCR4. Concurrently, endothelial cells produce PDGF $\beta$, whereas hepatic stellate cells express its receptor. SDF1 $\alpha$ and PDGF $\beta$ signaling maintain the close association between hepatic stellate cells and endothelial cells, which is critical for vascular tube formation and integrity. For hematopoietic stem cells (HSCs), hepatic stellate cells mediate their recruitment to the liver via SDF1 $\alpha /$ CXCR4 signaling. For hepatic epithelial cells, hepatic stellate cells regulate the proliferation of hepatoblast progenitor cells and hepatocytes by producing growth factors such as Wnt, FGF, HGF, and retinoic acid. They may also modulate the differentiation of hepatocytes and biliary cells from hepatoblasts by controlling the ECM composition within the liver. Lastly, hepatic stellate cells may contribute to the development of biliary cells by expressing the Notch ligand jagged 1 (Jag1).

both expressed in the septum transversum and hepatic stellate cells during development $(26,39)$. Wt1-null fetal livers show an abnormal increase of $\alpha$-SMA expression (40), suggestive of ectopic stellate cell activation. Similarly, $L h x 2$ knockout embryos contain numerous activated hepatic stellate cells and display a progressively increased deposition of ECM proteins associated with fibrosis (41). Therefore, despite being dispensable for hepatic stellate cell formation, both Wt1 and Lhx2 appear to keep these cells quiescent during development. The signal downstream of Wt 1 and Lhx 2 that prevents hepatic stellate cell activation is unclear. One candidate is the $\mathrm{Wnt} / \beta$-catenin pathway, as conditional deletion of $\beta$-catenin in the mesenchyme results in increased $\alpha$-SMA expression and ECM deposition in the liver $(42,43)$. On the other hand, freshly isolated hepatic stellate cells from adult mice exhibit hedgehog $(\mathrm{Hh})$ pathway activity, and inhibition of Hh signaling via pharmacologic inhibitor or neutralizing antibodies to Hh impairs hepatic stellate cell activation and decreases their survival (44). It will be interesting to investigate the role of the Hh pathway during the development of hepatic stellate cells.
Studies of the zebrafish hepatic stellate cell reporter line have shed light on the regulation of their differentiation and migration into the liver. Inhibition of VEGF signaling by global knockdown of VEGFR2 or by treatment with a VEGFR2 pharmacologic inhibitor during the course of hepatic stellate cell differentiation and migration drastically reduces their numbers (24). VEGF signaling does not appear to be essential for hepatic stellate cell survival, as blocking VEGFR2 during later stages only caused a moderate decrease in hepatic stellate cell numbers. Rather, VEGF may be required for hepatic stellate cell differentiation and/or their entry into the liver. Studies of liver injury and cancer have documented VEGF ligand expression by hepatocytes and biliary cells (45-47). Likewise, hepatic epithelial cells could be the source of VEGF for hepatic stellate cell development. Using an unbiased chemical screen approach, our group discovered two retinoid receptor agonists that have an opposing effect on hepatic stellate cell development (24). Compounds that modulate stellate cell differentiation, proliferation, or the switch between their quiescent and activated states during 


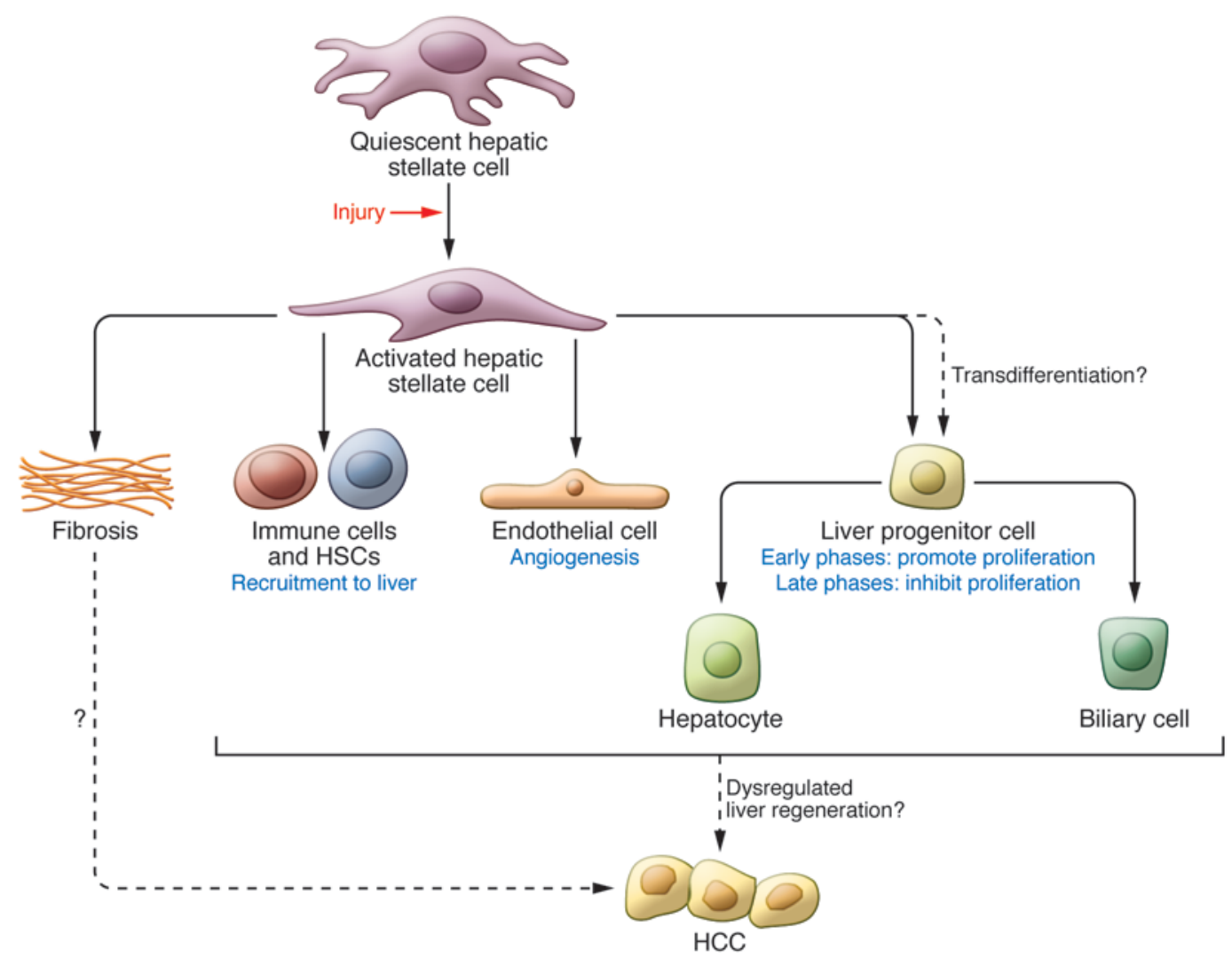

Figure 3

Hepatic stellate cells in liver regeneration and HCC. The biological processes that are influenced by hepatic stellate cells are indicated in blue. At early phases of liver regeneration, hepatic stellate cells promote the proliferation of liver progenitor cells and hepatocytes. They also stimulate angiogenesis in the wounded area and assist in the recruitment of hematopoietic stem cells and immune cells to the liver (reviewed in ref. 48). Recent studies suggest that activated hepatic stellate cells may undergo a mesenchymal-to-epithelial transition to transdifferentiate into liver progenitor cells. At late phases, hepatic stellate cells participate in the termination of regeneration, likely via high expression of TGF- $\beta$. Hepatic stellate cells have also been proposed to contribute to $\mathrm{HCC}$ development, potentially through dysregulation of some aspects of liver regeneration described above. On the other hand, liver fibrosis, which results from ectopic hepatic stellate cell activation, has controversial roles in HCC. Most evidence suggests that fibrosis promotes $\mathrm{HCC}$, but it is possible that in some clinical settings fibrosis and $\mathrm{HCC}$ might occur due to the same underlying factor(s) rather than one promoting the other.

development could potentially affect hepatic stellate cell behavior during injury, and thus have direct clinical implications.

The contribution of hepatic stellate cell to hepatic organogenesis. Throughout development, hepatic stellate cells are in close proximity to endothelial, hematopoietic, and hepatic epithelial cells, which suggests that hepatic stellate cells may modulate the growth, differentiation, or morphogenesis of these cells (summarized in Figure 2B). The interactions between stellate cells and other hepatic cells during development could be reactivated when the liver responds to injury.

Hepatic stellate cells contact sinusoidal endothelial cells by means of complex cytoplasmic processes, which ideally positions them for paracrine signaling with endothelial cells (48). During angiogenesis, interactions between pericytes and endothelial cells are essential for vascular tube maturation and integrity (49). Hepatic stellate cells are thought to be the pericyte equivalent in the liver and therefore may have the same impact on the development of the hepatic vasculature (50). In support of this notion, in mice that lack $\beta$-catenin in the liver mesenchyme, hepatic stellate cells become aberrantly activated and the liver is filled with dilated sinusoids (42).
During mammalian embryogenesis, the liver is the main site of hematopoiesis (51). In mice lacking the hepatic stellate cellexpressing homeobox gene $H l x$, fetal liver hematopoiesis is severely impaired (52), implicating hepatic stellate cells in this process. Fetal hepatic stellate cells express stromal cell-derived factor $1 \alpha$ (SDF1 $\alpha$; also known as CXCL12) (51), a potent chemoattractant for hematopoietic stem cells, which themselves express the SDF1 $\alpha$ receptor CXCR4 (53). Therefore, it is plausible that hepatic stellate cells are involved in recruiting hematopoietic stem/progenitor cells into the fetal liver.

Stellate cells first appear in mouse livers at around E10-E11, when differentiation of hepatocytes and biliary cells from hepatoblasts is still underway (54). Mouse fetal liver mesenchymal cells promote the maturation of hepatoblasts through cell-cell contact in cell culture (55). In Wt1 and Hlx mutant mice, the hepatoblast population fails to proliferate, resulting in smaller livers $(40,52)$. Fetal hepatic stellate cells express growth factors and mitogens such as Wnt9a (56), HGF (57), pleiotrophin (58), and FGF10 $(59,60)$, all of which have profound effects on the proliferation of hepatic epithelial cells during organ development and regeneration. In 
addition, hepatic stellate cells in the Wt1-null fetal livers show decreased expression of retinaldehyde dehydrogenase 2, an enzyme that catalyzes retinoic acid synthesis (40). The impairment of retinoic acid production could in turn affect hepatoblast proliferation. The role of hepatic stellate cells in hepatoblast differentiation is less clear. Nagai et al. reported that cell-cell contacts between hepatic stellate cells and hepatic epithelial cells induce the differentiation of the hepatocyte fate (61). On the other hand, the emergence and distribution of hepatic stellate cells also seem to correlate with the development of intrahepatic biliary cells (62). Hepatic stellate cells in rats express Notch receptors and target genes of Notch signaling (63), and Notch signaling plays key roles in the differentiation and morphogenesis of intrahepatic biliary cells (64). A recent study showed that inactivation of the Notch ligand jagged 1, which is expressed in the portal vein mesenchyme, leads to a paucity of intrahepatic bile ducts (65). Given that hepatic stellate cells also express jagged 1 (66), it will be interesting to investigate whether they modulate biliary cell development via Notch signaling. Alternatively, hepatic stellate cells could influence hepatoblast differentiation through production of ECM proteins, as different ECM components have different effects on the determination of the hepatocyte and biliary cell fate $(67,68)$.

The directed differentiation of human pluripotent stem cells into hepatocytes in culture could lead to new cell transplantation therapies for a wide range of acute and chronic liver diseases. Although important progress toward this goal has been made in recent years, liver cells differentiated in vitro do not share all the key characteristics of mature hepatocytes (reviewed in refs. 69, 70). Co-culturing primary human liver progenitor cells or hepatocytes with mesenchymal cells promotes or stabilizes hepatocyte differentiation (71-73). Therefore, understanding the interactions between hepatic stellate cells and hepatic epithelial cells during development is essential to create more efficient cell culture protocols for programmed differentiation of stem cells into hepatocytes.

\section{Hepatic stellate cells in liver regeneration}

Much as studies of liver development are highly relevant to creating new stem cell therapies, an understanding of liver regeneration has important implications for improving current methods of differentiating and propagating hepatocytes in vitro, as well as for stimulating hepatic recovery and improving survival after acute liver failure, liver transplantation, or resection. One of the oldest and most commonly used rodent models of liver regeneration is partial hepatectomy $(\mathrm{PH})$, in which two-thirds of the animal's liver is surgically removed $(74,75)$. Liver regeneration following $\mathrm{PH}$ is mainly driven by replication of existing hepatocytes and occurs in the absence of substantial necrosis and inflammation (74). To model how the liver regenerates when the ability of hepatocytes to divide is compromised, hepatocyte proliferation inhibitors such as 2-acetylaminofluorene can be administered before $\mathrm{PH}(2 \mathrm{AAF} / \mathrm{PH})$, which results in liver repopulation mediated by activation of liver progenitor cells or oval cells rather than proliferation of hepatocytes (74). Other rodent models of liver injury and regeneration involve chemical treatments with carbon tetrachloride $\left(\mathrm{CCl}_{4}\right)$ or acetaminophen (reviewed in ref. 76 ) or bile duct ligation (BDL) (77). While the PH model of liver regeneration may be particularly relevant to clinical scenarios in which the quantity of liver tissue is a limiting factor, such as small-for-size syndrome following liver transplantation, chemical injury and BDL models may more faithfully recapitulate the necrosis, inflammation, and/or fibrosis that accompany regeneration in chronic viral hepatitis, biliary tract disease, and/or drug-induced liver injury.

Activated hepatic stellate cells have been implicated in assisting liver regeneration by producing angiogenic factors as well as factors that modulate endothelial cell and hepatocyte proliferation and by remodeling the ECM (78). Recent evidence also suggests that in progenitor cell-mediated liver regeneration, hepatic stellate cells may, through a process of mesenchymal to epithelial transition, give rise to hepatocytes (21). Supporting the involvement of stellate cells in liver regeneration, inhibiting activated hepatic stellate cells using gliotoxin (79) and L-cysteine (80) prevents normal regenerative responses of both hepatocytes and oval cells in acetaminophen and $2 \mathrm{AAF} / \mathrm{PH}$-induced liver injuries, respectively. In addition, $\mathrm{Foxf1}^{+/-}$mice subjected to $\mathrm{CCl}_{4}$ injury show decreased hepatic stellate cell activation and more severe hepatocyte necrosis during the regenerative period (81). Notably, the mechanisms by which activated hepatic stellate cells help mediate liver regeneration in human patients and experimental animals remain to be determined and the relative importance of different subtypes of hepatic stellate cells/myofibroblasts is likely to depend on the nature of the initial insult.

Activated hepatic stellate cells produce a wide array of cytokines and chemokines (2). These factors may directly enhance the proliferation of liver progenitor cells and hepatocytes, or they may act indirectly through sinusoidal endothelial cells and immune cells to promote regeneration (ref. 2 and summarized in Figure 3). Conditioned media collected from hepatic stellate cells harvested from rats during early liver regeneration following $2 \mathrm{AAF} / \mathrm{PH}$ injury contain high levels of HGF and promote oval cell proliferation (82). One potential mediator of HGF production by hepatic stellate cells is the neurotrophin receptor P75 ${ }^{\mathrm{NTR}}$, which is expressed in human hepatic stellate cells following fibrotic liver injury. Murine hepatic stellate cells deficient for P75 ${ }^{\text {NTR }}$ do not differentiate properly into myofibroblasts in vitro or following liver injury induced by fibrin deposition in plasminogen-deficient $\left(\mathrm{Plg}^{-1-}\right)$ mice (83). Consequently, HGF production and hepatocyte proliferation are impaired in P75 ${ }^{N T R} ; P l g$ double-mutant mice (83). Hepatic stellate cell differentiation can be restored by constitutively active Rho in P75 ${ }^{\mathrm{NTR}}$-deficient hepatic stellate cells in vitro (83). These findings support a model in which $\mathrm{P} 75^{\mathrm{NTR}}$ promotes hepatic stellate cell activation via Rho, and activated stellate cells secrete HGF to stimulate hepatocyte proliferation during regeneration (83). Hh signaling is another important mediator of hepatic stellate cell-hepatocyte interactions during regeneration. Culture-activated hepatic stellate cells synthesize sonic hedgehog (Shh), which serves as an autocrine growth factor for these cells (84). In vivo, Hh ligands induce hepatocyte proliferation after $\mathrm{PH}(85)$.

Notably, activated hepatic stellate cells are the main source of matrix metalloproteinases and their inhibitors that participate in ECM remodeling. The production of cytokines and remodeling of the ECM are likely to be coupled, as the ECM is capable of sequestering biologically active molecules $(86,87)$. Thus in addition to directly secreting cytokines, activated hepatic stellate cells may modulate their function by cleaving or releasing cytokines from the ECM.

Liver regeneration is a multistep process involving both initiation and termination of liver growth. The liver stops regenerating when it attains the mass required for the needs of the organism (88). The most well-known hepatocyte antiproliferative factor is 
TGF- $\beta$, and one of the primary TGF- $\beta$-producing cell types in the liver are hepatic stellate cells (89). How do hepatic stellate cells mediate both the initiation and cessation of liver regeneration? As mentioned earlier, conditioned medium collected from hepatic stellate cells at early phases of liver regeneration in a $2 \mathrm{AAF} / \mathrm{PH}$ injury model contains high levels of HGF. This strong mitogen may override the antiproliferative effect of TGF- $\beta 1$ (82). In contrast, at terminal phases of liver regeneration, hepatic stellate cells produce high levels of TGF- $\beta 1$, which inhibits hepatocyte proliferation and even induces apoptosis. Serotonin has been shown to increase expression of TGF- $\beta 1$ in cultured primary mouse hepatic stellate cells via the 5-hydroxytryptamine $2 \mathrm{~B}\left(5-\mathrm{HT}_{2 \mathrm{~B}}\right)$ receptor, and $5-\mathrm{HT}_{2 \mathrm{~B}}$ inhibition promotes hepatocyte proliferation following $\mathrm{PH}, \mathrm{BDL}$, and $\mathrm{CCl}_{4}$-induced liver injury (90). Thus, hepatic stellate cells may change their cytokine expression profile during the process of liver regeneration, regulating both its initiation and termination.

To fully characterize the role of hepatic stellate cells in liver regeneration, their specific ablation would be highly useful, ideally at different time points in the regenerative process. While some chemical tools, including gliotoxin (79) or L-cysteine (80), exist for selective inhibition of hepatic stellate cells in rodent models, the possibility that these drugs also affect other hepatic cell types is difficult to exclude. A recent study indicates that hepatic stellate cells can be depleted in mice by using the GFAP promoter to drive the herpes simplex virus-thymidine kinase gene expression, rendering proliferating hepatic stellate cells susceptible to gancyclovir-induced death (20). An advantage of this new model is the ability to target proliferating hepatic stellate cells in vivo without affecting quiescent hepatic stellate cells or other myofibroblasts. However, hepatic stellate cells cannot be completely ablated using this model, as GFAP is not universally expressed in these cells.

Any single animal model is unlikely to completely mimic all relevant aspects of human liver regeneration, particularly given that the cellular and molecular pathways mediating regeneration are likely to vary somewhat depending on the nature of the initial injury. Therefore, future studies of hepatic stellate cells in liver regeneration will be facilitated by the availability of multiple animal models, which are likely to yield complementary insights. Advantages of rodent models include the ability to isolate, culture, and activate hepatic stellate cells in vitro, facilitating follow-up cell culture studies focused on molecular mechanisms involved in regeneration. On the other hand, the excellent live-imaging technologies available in zebrafish are well suited for studying the cellular interactions at play during the regenerative process. As with rodents, $\mathrm{PH}$ or toxic chemicals can be used to induce liver regeneration in zebrafish (reviewed in ref. 74). Genetic tools have enabled the development of additional regeneration models including the nitroreductase/ metronidazole cell ablation system (91) and morpholino-based knockdown of a mitochondrial import gene to induce hepatocyte death (92). One promising approach is to perform high-throughput chemical screens in various zebrafish models of liver injury, seeking drugs that affect stellate cells during liver regeneration (24).

\section{Hepatic stellate cells in cancer}

While promotion of hepatocyte proliferation and liver regeneration may be desirable in some clinical settings, aberrant activation of such processes can also be associated with human diseases, most notably HCC (summarized in Figure 3). The majority of human HCCs occur in the setting of clinically significant fibrosis or cir- rhosis (93), implicating hepatic stellate cells in their pathogenesis as the major ECM-producing cell type of the liver. The associations between HCC and fibrosis are incompletely understood, but likely involve inflammatory cells, integrin signaling, growth factor interactions with the ECM, and communication between activated hepatic stellate cells and tumor cells (reviewed in ref. 94). Activated hepatic stellate cells are present between endothelial cells and cancer cell trabeculae in patients with HCC (95), and conditioned media from activated hepatic stellate cells increases proliferation and migration of human HCC cells (96). Thus, most evidence suggests that fibrosis promotes HCC, but it is possible that in some clinical settings fibrosis and HCC might occur due to the same underlying factor(s) rather than one promoting the other.

Chemical compounds such as $\mathrm{N}$-nitrosodiethylamine, $\mathrm{CCl}_{4}$, and aflatoxin $\mathrm{B}_{1}$ cause $\mathrm{HCC}$ in rodents that is preceded by chronic liver injury, mimicking the injury-fibrosis-malignancy sequence that characterizes most human HCCs (97). However, tumor phenotypes in these models are dependent on animal age, strain, and the route of drug administration, and tumor latency can be quite long (97). On the other hand, liver tumors induced genetically in mice via expression of growth factors such as TGF- $\alpha$, oncogenes such as Myc, and viral proteins such as $\mathrm{HB}_{\mathrm{X}}$ are more tractable but are not usually preceded by substantial fibrosis $(98,99)$. Thus, the opportunity for studying hepatic stellate cell-HCC interactions in transgenic mouse models of HCC has been somewhat limited, with the notable exception of the PDGF-C transgenic mouse (100). These mice, whose hepatocytes express human PDGF-C, show hepatic stellate cell activation and collagen deposition followed by hepatomegaly and HCC. These in vivo findings correlate with in vitro studies demonstrating that PDGF-C promotes the proliferation, survival, and migration of fibroblasts and pericytes (101).

Interactions between hepatic stellate cells and HCC cells in vivo have also been studied by co-transplanting hepatic stellate cells and malignant hepatocytes into immunocompromised mice. These studies have implicated TGF- $\beta$ signaling $(102,103)$ and regulatory $\mathrm{T}$ cells (104) as mechanisms by which hepatic stellate cells may promote HCC growth. On the other hand, experiments performed in lecithin retinol acyltransferase-deficient mice have revealed ways by which HCC growth might be inhibited via targeting of hepatic stellate cells $(105,106)$. These mice lack retinoidcontaining lipid droplets in hepatic stellate cells, exhibit increased retinoic acid signaling, and show decreased tumor formation in response to diethylnitrosamine, suggesting that altering retinoic acid signaling in stellate cells may inhibit HCC growth.

Zebrafish develop liver tumors that are morphologically and genetically similar to human HCC (107-110). Similar to many rodent models, zebrafish HCC models are not typically preceded by cirrhosis, although co-expression of hepatitis B virus $\mathrm{X}$ and hepatitis $\mathrm{C}$ virus core proteins in zebrafish liver leads to fibrosis and cholangiocarcinoma (111). This model may thus be useful to study hepatic stellate cell interactions with primary liver tumor cells in vivo.

While many pathways that mediate hepatic stellate cell-HCC interactions have been implicated (reviewed in ref. 94), the effects of specifically inhibiting or activating these pathways in vivo have not been fully explored. Driving expression of candidate positive or negative regulators specifically in hepatic stellate cells or creating stellate cell-specific gene knockouts could be useful in this regard. A major challenge for these experiments, as in studies of hepatic stellate cell development, is the identification of promoters with improved specificity. Similarly, improved techniques for 
ablating or inhibiting hepatic stellate cells could help tease out the role of these cells at different time points in HCC formation. Such studies could help define when and how hepatic stellate cells could be targeted to prevent or treat HCC.

A more efficient way to detect HCC could profoundly improve prognosis by enabling earlier diagnosis and more effective treatments. New HCC biomarkers that have been proposed include molecules produced by hepatic stellate cells, such as HGF and IGF (112). Patients with HCC also show elevated plasma levels of TGF- $\beta 1$ (113) and osteopontin (114), compared with patients with chronic hepatitis and/or cirrhosis. As many of the same factors are produced by hepatic stellate cells during cirrhosis and during carcinogenesis, it is likely that a combination of biomarkers will be required to optimize early $\mathrm{HCC}$ detection.

\section{Conclusions}

Studies of hepatic stellate cell behavior during development, regeneration, and tumor formation using cell culture and animal models have provided substantial insights regarding the cellular and molecular mechanisms involved in these processes. It will be crucial to identify promoters with improved cell type specificity, as they will facilitate hepatic stellate cell-specific manipulations, including gene knockouts and cell ablation. Given the critical roles that hepatic stellate cells play in diverse aspects of liver pathophysiology, this intriguing cell type represents a major, and mostly untapped, potential reservoir for the development of therapies targeting a wide variety of human liver diseases, ranging from acute liver failure to drug-induced liver injury to HCC.

1. Blaner WS, et al. Hepatic stellate cell lipid droplets: a specialized lipid droplet for retinoid storage. Biochim Biophys Acta. 2009;1791(6):467-473.

2. Friedman SL. Hepatic stellate cells: protean, multifunctional, and enigmatic cells of the liver. Physiol Rev. 2008;88(1):125-172.

3. Lee UE, Friedman SL. Mechanisms of hepatic fibrogenesis. Best Pract Res Clin Gastroenterol. 2011; 25(2):195-206.

4. Ellis EL, Mann DA. Clinical evidence for the regression of liver fibrosis. J Hepatol. 2012;56(5):1171-1180.

5. Kisseleva T, et al. Myofibroblasts revert to an inactive phenotype during regression of liver fibrosis. Proc Natl Acad Sci U S A. 2012;109(24):9448-9453.

6. Troeger JS, et al. Deactivation of hepatic stellate cells during liver fibrosis resolution in mice. Gastroenterology. 2012;143(4):1073-1083 e1022

7. Krizhanovsky V, et al. Senescence of activated stellate cells limits liver fibrosis. Cell. 2008;134(4):657-667.

8. Schuppan D, Kim YO. Evolving therapies for liver fibrosis. J Clin Invest. 2013;123(5):1887-1901.

9. Bachem MG, Meyer D, Melchior R, Sell KM, Gressner AM. Activation of rat liver perisinusoidal lipocytes by transforming growth factors derived from myofibroblastlike cells. A potential mechanism of self perpetuation in liver fibrogenesis. J Clin Invest. 1992;89(1):19-27.

10. Friedman SL, Roll FJ, Boyles J, Bissell DM. Hepatic lipocytes: the principal collagen-producing cells of normal rat liver. Proc Natl Acad Sci U S A. 1985; 82(24):8681-8685.

11. Friedman SL, Roll FJ, Boyles J, Arenson DM, Bissell DM. Maintenance of differentiated phenotype of cultured rat hepatic lipocytes by basement membrane matrix. J Biol Chem. 1989;264(18):10756-10762.

12. Greenwel P, Schwartz M, Rosas M, Peyrol S, Grimaud JA, Rojkind M. Characterization of fat-storing cell lines derived from normal and CCl4-cirrhotic livers. Differences in the production of interleukin-6. Lab Invest. 1991;65(6):644-653.

13. Sauvant P, et al. PAV-1, a new rat hepatic stellate

\section{Acknowledgments}

The authors thank Jacquelyn Maher for her critical comments and support. C. Yin is supported by grant K99AA020514 from the NIH and the University of California San Francisco Liver Center Pilot/Feasibility Award (NIH grant P30DK026743). K.J. Evason is a Robert Black Fellow supported by the Damon Runyon Cancer Research Foundation (grant DRG-109-10). K. Asahina is supported by a grant from the NIH (R01AA020753). Our work on hepatic stellate cells and liver development was further supported by grants from the NIH (R01DK060322) and the Packard Foundation (to D.Y.R. Stainer).

Address correspondence to: Chunyue Yin, Cincinnati Children's Hospital Medical Center, Gastroenterology, Hepatology and Nutrition Division, 3333 Burnet Avenue, Cincinnati, Ohio 45229, USA. Phone: 513.803.8096. Fax: 513.636.5581. E-mail: chunyue. yin@cchmc.org Or to: Didier Y.R. Stainier, Department of Developmental Genetics, Max Planck Institute for Heart and Lung Research, Bad Nauheim, Germany. Phone: 49.6032.705.1301. Fax: 49.6032.705.1304. Email: stainier@mpg-bn.mpg.de.

Chunyue Yin's present address is: Division of Gastroenterology, Hepatology and Nutrition, Cincinnati Children's Hospital Medical Center, Cincinnati, Ohio, USA.

Didier Y.R. Stainier's present address is: Department of Developmental Genetics, Max Planck Institute for Heart and Lung Research, Bad Nauheim, Germany. cell line converts retinol into retinoic acid, a process altered by ethanol. Int J Biochem Cell Biol. 2002; 34(8):1017-1029.

14. Xu L, et al. Human hepatic stellate cell lines, LX-1 and LX-2: new tools for analysis of hepatic fibrosis. Gut. 2005;54(1):142-151.

15. De Minicis S, et al. Gene expression profiles during hepatic stellate cell activation in culture and in vivo. Gastroenterology. 2007;132(5):1937-1946.

16. Yokoi Y, et al. Immunocytochemical detection of desmin in fat-storing cells (Ito cells). Hepatology. 1984;4(4):709-714.

17. Gard AL, White FP, Dutton GR. Extra-neural glial fibrillary acidic protein (GFAP) immunoreactivity in perisinusoidal stellate cells of rat liver. J Neuroimmunol. 1985;8(4-6):359-375.

18. Ramadori $\mathrm{G}$, et al. Expression of the gene of the alpha-smooth muscle-actin isoform in rat liver and in rat fat-storing (ITO) cells. Virchows Arch B Cell Pathol Incl Mol Pathol. 1990;59(6):349-357.

19. Magness ST, Bataller R, Yang L, Brenner DA. A dual reporter gene transgenic mouse demonstrates heterogeneity in hepatic fibrogenic cell populations. Hepatology. 2004;40(5):1151-1159.

20. Puche JE, et al. A novel murine model to deplete hepatic stellate cells uncovers their role in amplifying liver damage. Hepatology. 2013;57(1):339-350.

21. Yang L, et al. Fate-mapping evidence that hepatic stellate cells are epithelial progenitors in adult mouse livers. Stem Cells. 2008;26(8):2104-2113.

22. Chu J, Sadler KC. New school in liver development: lessons from zebrafish. Hepatology. 2009; 50(5):1656-1663

23. Beis D, Stainier DY. In vivo cell biology: following the zebrafish trend. Trends Cell Biol. 2006; 16(2):105-112.

24. Yin C, Evason KJ, Maher JJ, Stainier DY. The bHLH transcription factor Hand 2 marks hepatic stellate cells in zebrafish: Analysis of stellate cell entry into the developing liver. Hepatology. 2012;56(5):1958-1970.

25. Asahina K, et al. Mesenchymal origin of hepatic stellate cells, submesothelial cells, and perivascular mesenchymal cells during mouse liver development. Hepatology. 2009;49(3):998-1011.

26. Asahina K, Zhou B, Pu WT, Tsukamoto H. Septum transversum-derived mesothelium gives rise to hepatic stellate cells and perivascular mesenchymal cells in developing mouse liver. Hepatology. 2011; 53(3):983-995.

27. Geerts A. On the origin of stellate cells: mesodermal, endodermal or neuro-ectodermal? J Hepatol. 2004; 40(2):331-334.

28. Suskind DL, Muench MO. Searching for common stem cells of the hepatic and hematopoietic systems in the human fetal liver: CD34+ cytokeratin 7/8+ cells express markers for stellate cells. J Hepatol. 2004;40(2):261-268.

29. Sicklick JK, et al. Evidence for epithelial-mesenchymal transitions in adult liver cells. Am J Physiol Gastrointest Liver Physiol. 2006;291(4):G575-G583.

30. Wells RG. The epithelial-to-mesenchymal transition in liver fibrosis: here today, gone tomorrow? Hepatology. 2010;51(3):737-740.

31. Miyata E, et al. Hematopoietic origin of hepatic stellate cells in the adult liver. Blood. 2008; 111(4):2427-2435.

32. Baba S, et al. Commitment of bone marrow cells to hepatic stellate cells in mouse. J Hepatol. 2004; 40(2):255-260.

33. Higashiyama R, et al. Negligible contribution of bone marrow-derived cells to collagen production during hepatic fibrogenesis in mice. Gastroenterology. 2009;137(4):1459-1466 e1451.

34. Kisseleva T, Brenner DA. The phenotypic fate and functional role for bone marrow-derived stem cells in liver fibrosis. J Hepatol. 2011;56(4):965-972.

35. Knittel T, et al. Localization of liver myofibroblasts and hepatic stellate cells in normal and diseased rat livers: distinct roles of (myo-)fibroblast subpopulations in hepatic tissue repair. Histochem Cell Biol. 1999;112(5):387-401.

36. Knittel T, et al. Rat liver myofibroblasts and 
hepatic stellate cells: different cell populations of the fibroblast lineage with fibrogenic potential. Gastroenterology. 1999;117(5):1205-1221.

37. Ankoma-Sey V, et al. Coordinated induction of VEGF receptors in mesenchymal cell types during rat hepatic wound healing. Oncogene. 1998; 17(1):115-121.

38. Perez-Pomares JM, Carmona R, Gonzalez-Iriarte M, Macias D, Guadix JA, Munoz-Chapuli R. Contribution of mesothelium-derived cells to liver sinusoids in avian embryos. Dev Dyn. 2004;229(3):465-474.

39. Kolterud A, Wandzioch E, Carlsson L. Lhx2 is expressed in the septum transversum mesenchyme that becomes an integral part of the liver and the formation of these cells is independent of functional Lhx2. Gene Expr Patterns. 2004;4(5):521-528.

40. Ijpenberg A, et al. Wt 1 and retinoic acid signaling are essential for stellate cell development and liver morphogenesis. Dev Biol. 2007;312(1):157-170.

41. Wandzioch E, Kolterud A, Jacobsson M, Friedman SL, Carlsson L. Lhx2 $2^{-/-}$mice develop liver fibrosis. Proc Natl Acad Sci US A. 2004;101(47):16549-16554.

42. Berg T, DeLanghe S, Al Alam D, Utley S, Estrada J, Wang KS. beta-catenin regulates mesenchymal progenitor cell differentiation during hepatogenesis. J Surg Res. 2010;164(2):276-285.

43. Kordes C, Sawitza I, Haussinger D. Canonical Wnt signaling maintains the quiescent stage of hepatic stellate cells. Biochem Biophys Res Commun. 2008; 367(1):116-123.

44. Sicklick JK, et al. Role for hedgehog signaling in hepatic stellate cell activation and viability. Lab Invest. 2005;85(11):1368-1380.

45. Mochida S, Ishikawa K, Inao M, Shibuya M, Fujiwara K. Increased expressions of vascular endothelial growth factor and its receptors, flt-1 and $\mathrm{KDR} / \mathrm{flk}-1$, in regenerating rat liver. Biochem Biophys Res Commun. 1996;226(1):176-179.

46. Suzuki K, et al. Expression of vascular permeability factor/vascular endothelial growth factor in human hepatocellular carcinoma. Cancer Res. 1996; 56(13):3004-3009

47. Ogasawara $S$, et al. Expression of angiogenic factors, basic fibroblast growth factor and vascular endothelial growth factor, in human biliary tract carcinoma cell lines. Hepatol Res. 2001;20(1):97-113.

48. Lee JS, Semela D, Iredale J, Shah VH. Sinusoidal remodeling and angiogenesis: a new function for the liver-specific pericyte? Hepatology. 2007; 45(3):817-825.

49. Hellstrom M, Kalen M, Lindahl P, Abramsson A, Betsholtz C. Role of PDGF-B and PDGFR-beta in recruitment of vascular smooth muscle cells and pericytes during embryonic blood vessel formation in the mouse. Development. 1999; 126(14):3047-3055.

50. Semela D, Das A, Langer D, Kang N, Leof E, Shah V. Platelet-derived growth factor signaling through ephrin-b2 regulates hepatic vascular structure and function. Gastroenterology. 2008;135(2):671-679.

51. Kubota H, Yao HL, Reid LM. Identification and characterization of vitamin A-storing cells in fetal liver: implications for functional importance of hepatic stellate cells in liver development and hematopoiesis. Stem Cells. 2007;25(9):2339-2349.

52. Hentsch B, et al. Hlx homeo box gene is essential for an inductive tissue interaction that drives expansion of embryonic liver and gut. Genes Dev. 1996; 10(1):70-79

53. Wright DE, Bowman EP, Wagers AJ, Butcher EC, Weissman IL. Hematopoietic stem cells are uniquely selective in their migratory response to chemokines. J Exp Med. 2002;195(9):1145-1154.

54. Lemaigre FP. Mechanisms of liver development: concepts for understanding liver disorders and design of novel therapies. Gastroenterology. 2009; 137(1):62-79.

55. Hoppo T, et al. Thy1-positive mesenchymal cells promote the maturation of CD49f-positive hepatic progenitor cells in the mouse fetal liver. Hepatology. 2004;39(5):1362-1370.

56. Matsumoto K, Miki R, Nakayama M, Tatsumi $\mathrm{N}$, Yokouchi Y. Wnt9a secreted from the walls of hepatic sinusoids is essential for morphogenesis, proliferation, and glycogen accumulation of chick hepatic epithelium. Dev Biol. 2008;319(2):234-247.

57. Maher JJ. Cell-specific expression of hepatocyte growth factor in liver. Upregulation in sinusoidal endothelial cells after carbon tetrachloride. J Clin Invest. 1993;91(5):2244-2252.

58. Asahina K, et al. Pleiotrophin/heparin-binding growth-associated molecule as a mitogen of rat hepatocytes and its role in regeneration and development of liver. Am J Pathol. 2002;160(6):2191-2205.

59. Sekhon SS, Tan X, Micsenyi A, Bowen WC, Monga SP. Fibroblast growth factor enriches the embryonic liver cultures for hepatic progenitors. Am J Pathol. 2004;164(6):2229-2240.

60 . Berg T, et al. Fibroblast growth factor 10 is critical for liver growth during embryogenesis and controls hepatoblast survival via beta-catenin activation. Hepatology. 2007;46(4):1187-1197.

61. Nagai H, et al. Differentiation of liver epithelial (stem-like) cells into hepatocytes induced by coculture with hepatic stellate cells. Biochem Biophys Res Commun. 2002;293(5):1420-1425.

62. Libbrecht L, Cassiman D, Desmet V, Roskams T. The correlation between portal myofibroblasts and development of intrahepatic bile ducts and arterial branches in human liver. Liver. 2002;22(3):252-258.

63. Sawitza I, Kordes C, Reister S, Haussinger D. The niche of stellate cells within rat liver. Hepatology. 2009;50(5):1617-1624.

64. Zong Y, et al. Notch signaling controls liver development by regulating biliary differentiation. Development. 2009;136(10):1727-1739.

65. Hofmann JJ, Zovein AC, Koh H, Radtke F, Weinmaster G, Iruela-Arispe ML. Jagged 1 in the portal vein mesenchyme regulates intrahepatic bile duct development: insights into Alagille syndrome. Development. 2010;137(23):4061-4072.

66. Suzuki K, Tanaka M, Watanabe N, Saito S, Nonaka H, Miyajima A. p75 Neurotrophin receptor is a marker for precursors of stellate cells and portal fibroblasts in mouse fetal liver. Gastroenterology. 2008;135(1):270-281.

67. Suzuki A, Iwama A, Miyashita H, Nakauchi H, Taniguchi $\mathrm{H}$. Role for growth factors and extracellular matrix in controlling differentiation of prospectively isolated hepatic stem cells. Development. 2003; 130(11):2513-2524.

68. Yanai M, Tatsumi N, Hasunuma N, Katsu K, Endo F, Yokouchi Y. FGF signaling segregates biliary celllineage from chick hepatoblasts cooperatively with BMP4 and ECM components in vitro. Dev Dyn. 2008;237(5):1268-1283.

69. Chistiakov DA. Liver regenerative medicine: advances and challenges. Cells Tissues Organs. 2012; 196(4):291-312.

70. Rountree CB, Mishra L, Willenbring H. Stem cells in liver diseases and cancer: recent advances on the path to new therapies. Hepatology. 2012;55(1):298-306.

71. Wang Y, et al. Paracrine signals from mesenchymal cell populations govern the expansion and differentiation of human hepatic stem cells to adult liver fates. Hepatology. 2010;52(4):1443-1454.

72. Okamoto M, Ishida Y, Keogh A, Strain A. Evaluation of the function of primary human hepatocytes cocultured with the human hepatic stellate cell (HSC) line LI90. Int J ArtifOrgans. 1998;21(6):353-359.

73. Mitaka T, Sato F, Mizuguchi T, Yokono T, Mochizuki Y. Reconstruction of hepatic organoid by rat small hepatocytes and hepatic nonparenchymal cells. Hepatology. 1999;29(1):111-125.

74. Curado S, Stainier DY. deLiver'in regeneration: injury response and development. Semin Liver Dis.
2010;30(3):288-295

75. Rutherford A, Chung RT. Acute liver failure: mechanisms of hepatocyte injury and regeneration. Semin Liver Dis. 2008;28(2):167-174.

76. Tunon MJ, Alvarez M, Culebras JM, Gonzalez-Gallego J. An overview of animal models for investigating the pathogenesis and therapeutic strategies in acute hepatic failure. World J Gastroenterol. 2009; 15(25):3086-3098.

77. Alatas FS, et al. Synchronized expressions of hepatic stellate cells and their transactivation and liver regeneration during liver injury in an animal model of cholesatsis. J Pediatr Surg. 2011; 46(12):2284-2290.

78. Roskams T. Relationships among stellate cell activation, progenitor cells, and hepatic regeneration. Clin Liver Dis. 2008;12(4):853-860, ix

79. Shen K, et al. Depletion of activated hepatic stellate cell correlates with severe liver damage and abnormal liver regeneration in acetaminophen-induced liver injury. Acta Biochim Biophys Sin (Shanghai). 2011; 43(4):307-315.

80. Pintilie DG, Shupe TD, Oh SH, Salganik SV, Darwiche $\mathrm{H}$, Petersen BE. Hepatic stellate cells' involvement in progenitor-mediated liver regeneration. Lab Invest. 2010;90(8):1199-1208.

81. Kalinichenko VV, et al. Foxf1 $1^{+/-}$mice exhibit defective stellate cell activation and abnormal liver regeneration following CCl4 injury. Hepatology. 2003; 37(1):107-117.

82. Chen L, et al. HSCs play a distinct role in different phases of oval cell-mediated liver regeneration. Cell Biochem Funct. 2012;30(7):588-596.

83. Passino MA, Adams RA, Sikorski SL, Akassoglou K. Regulation of hepatic stellate cell differentiation by the neurotrophin receptor p75NTR. Science. 2007; 315(5820):1853-1856.

84. Yang $\mathrm{L}$, et al. Sonic hedgehog is an autocrine viability factor for myofibroblastic hepatic stellate cells. J Hepatol. 2008;48(1):98-106.

85. Ochoa B, et al. Hedgehog signaling is critical for normal liver regeneration after partial hepatectomy in mice. Hepatology. 2010;51(5):1712-1723.

86. Pi L, et al. Connective tissue growth factor with a novel fibronectin binding site promotes cell adhesion and migration during rat oval cell activation. Hepatology. 2008;47(3):996-1004.

87. Schuppan D, et al. Collagens in the liver extracellular matrix bind hepatocyte growth factor. Gastroenterology. 1998;114(1):139-152.

88. Taub R. Liver regeneration: from myth to mechanism. Nat Rev Mol Cell Biol. 2004;5(10):836-847.

89. Karkampouna S, Ten Dijke P, Dooley S, Julio MK. TGFbeta Signaling in Liver Regeneration. Curr Pharm Des. 2012;18(27):4103-4113.

90. Ebrahimkhani MR, et al. Stimulating healthy tissue regeneration by targeting the 5-HT(2)B receptor in chronic liver disease. Nat Med. 2011; 17(12):1668-1673.

91. Curado S, Anderson RM, Jungblut B, Mumm J, Schroeter E, Stainier DY. Conditional targeted cell ablation in zebrafish: a new tool for regeneration studies. Dev Dyn. 2007;236(4):1025-1035.

92. Curado $S$, et al. The mitochondrial import gene tomm 22 is specifically required for hepatocyte survival and provides a liver regeneration model. Dis Model Mech. 2010;3(7-8):486-495

93. Seitz HK, Stickel F. Risk factors and mechanisms of hepatocarcinogenesis with special emphasis on alcohol and oxidative stress. Biol Chem. 2006; 387(4):349-360.

94. Zhang DY, Friedman SL. Fibrosis-dependent mechanisms of hepatocarcinogenesis. Hepatology. 2012; 56(2):769-775

95. Enzan $\mathrm{H}$, et al. Alpha-smooth muscle actin-positive perisinusoidal stromal cells in human hepatocellular carcinoma. Hepatology. 1994;19(4):895-903.

96. Amann T, et al. Activated hepatic stellate cells pro- 
mote tumorigenicity of hepatocellular carcinoma. Cancer Sci. 2009;100(4):646-653.

97. Heindryckx F, Colle I, Van Vlierberghe H. Experimental mouse models for hepatocellular carcinoma research. Int J Exp Pathol. 2009;90(4):367-386.

98. Fausto N, Campbell JS. Mouse models of hepatocellular carcinoma. Semin Liver Dis. 2010;30(1):87-98.

99. Li Y, Tang ZY, Hou JX. Hepatocellular carcinoma: insight from animal models. Nat Rev Gastroenterol Hepatol. 2012;9(1):32-43.

100.Campbell JS, et al. Platelet-derived growth factor $\mathrm{C}$ induces liver fibrosis, steatosis, and hepatocellular carcinoma. Proc Natl Acad Sci U S A. 2005; 102(9):3389-3394.

101. Hou X, et al. PDGF-CC blockade inhibits pathological angiogenesis by acting on multiple cellular and molecular targets. Proc Natl Acad Sci U S A. 2010; 107(27):12216-12221.

102. Mikula M, Proell V, Fischer AN, Mikulits W. Activated hepatic stellate cells induce tumor progression of neoplastic hepatocytes in a TGF-beta dependent fashion. J Cell Physiol. 2006;209(2):560-567. 103.van Zijl F, et al. Hepatic tumor-stroma crosstalk guides epithelial to mesenchymal transition at the tumor edge. Oncogene. 2009;28(45):4022-4033.

104.Zhao W, et al. Activated hepatic stellate cells promote hepatocellular carcinoma development in immunocompetent mice. Int J Cancer. 2011; 129(11):2651-2661.

105. Kluwe J, et al. Absence of hepatic stellate cell retinoid lipid droplets does not enhance hepatic fibrosis but decreases hepatic carcinogenesis. Gut. 2011; 60(9):1260-1268.

106. Shirakami Y, Gottesman ME, Blaner WS. Diethylnitrosamine-induced hepatocarcinogenesis is suppressed in lecithin:retinol acyltransferase-deficient mice primarily through retinoid actions immediately after carcinogen administration. Carcinogenesis. 2012;33(2):268-274.

107. Huang X, Zhou L, Gong Z. Liver tumor models in transgenic zebrafish: an alternative in vivo approach to study hepatocarcinogenes. Future Oncol. 2012;8(1):21-28.

108.Liu S, Leach SD. Zebrafish models for cancer. Annu
Rev Pathol. 2011;6:71-93.

109. Goessling W, North TE, Zon LI. New waves of discovery: modeling cancer in zebrafish.J Clin Oncol. 2007; 25(17):2473-2479.

110. Mudbhary R, Sadler KC. Epigenetics, development, and cancer: zebrafish make their mark. Birth Defects Res C Embryo Today. 2011;93(2):194-203.

111. Liu W, et al. A zebrafish model of intrahepatic cholangiocarcinoma by dual expression of hepatitis $B$ virus $X$ and hepatitis $C$ virus core protein in liver. Hepatology. 2012;56(6):2268-2276.

112. Bertino G, Ardiri A, Malaguarnera M, Malaguarnera G, Bertino N, Calvagno GS. Hepatocellualar carcinoma serum markers. Semin Oncol. 2012; 39(4):410-433.

113. Shirai Y, et al. Plasma transforming growth factor-beta 1 in patients with hepatocellular carcinoma. Comparison with chronic liver diseases. Cancer. 1994;73(9):2275-2279.

114.Shang $\mathrm{S}$, et al. Identification of osteopontin as a novel marker for early hepatocellular carcinoma. Hepatology. 2012;55(2):483-490. 